\title{
Electrophysiological features in patients with sinus node dysfunction and vasovagal syncope
}

Beata Graff', Grzegorz Graff², Edward Koźluk³ , Monika Tokarczyk4, Agnieszka Piątkowska³ Szymon Budrejko ${ }^{4}$, Dariusz Kozłowski ${ }^{4}$, Alicja Dąbrowska-Kugacka ${ }^{4}$, Ewa Lewicka ${ }^{4}$, Grażyna Świątecka ${ }^{4}$, Grzegorz Raczak ${ }^{4}$

${ }^{1}$ Hypertension Unit, Department of Hypertension and Diabetology, Medical University of Gdansk, Poland

2Faculty of Applied Physics and Mathematics, Gdansk University of Technology,

Poland

${ }^{3} 1^{\text {st }}$ Chair and Department of Cardiology, Medical University of Warsaw, Poland

${ }^{4}$ Department of Cardiology and Electrotherapy, Medical University of Gdansk, Poland

Submitted: 25 June 2010

Accepted: 11 April 2011

Arch Med Sci 2011; 7, 6: 963-970

DOI: 10.5114/AOMS.2011.26607

Copyright () 2011 Termedia \& Banach

\section{Abstract}

Introduction: Syncope is a common presentation of sinus node dysfunction (SND). Some patients who receive a permanent pacemaker due to SND do not benefit from it and further diagnostic workup leads to the diagnosis of vasovagal syncope (VVS). The aim of the study was to identify electrophysiological criteria that can be used for identification of patients with SND and concurrent VVS.

Material and methods: Transoesophageal atrial pacing (TAP) was performed in 100 patients divided into four groups depending on symptoms and TAP results. Standard electrophysiological parameters of sinus node function and their variability were obtained in the basal state and after pharmacological autonomic blockade $(A B)$.

Results: Patients with concurrent SND and VVS had a greater variability of sinoatrial conduction time assessed by Strauss' method than patients without incidents of syncope ( $83.2 \pm 53.9$ vs. $34.1 \pm 19.6,47.8 \pm 33.6$ and $32.1 \pm 22.99)$. Apart from abnormal sinus node recovery time and second pause, patients with SND had bigger basal state variability of these parameters. In patients with SND and concurrent vasovagal syncope the variability of sinus node recovery time (SNRT), corrected SNRT (cSNRT) and second pause (IIP) decreased after autonomic blockade.

Conclusions: Patients with concurrent SND and VVS have distinct electrophysiological features - greater sinoatrial conduction time (SACT) variability and the decrease of SNRT, CSNRT and IIP variability after AB. However, further studies in larger study groups are needed to validate our findings. Transoesophageal atrial pacing is a useful procedure in patients with syncope, especially when the coexistence of more than one cardiac cause is suspected.

Key words: syncope, sinus node dysfunction, vasovagal syncope, transoesophageal atrial pacing, sinoatrial conduction time variability.

\section{Introduction}

Syncope is a common presentation of sinus node dysfunction. However, there are no exact values of electrophysiological parameters which can be useful to discriminate whether sinus node dysfunction is the cause of syncope. The sensitivity of sinus node recovery time (SNRT) or corrected

\author{
Corresponding author: \\ Dariusz Kozłowski MD, PhD \\ Department of Cardiology \\ and Electrotherapy \\ Medical University of Gdansk \\ 7 Debinki St \\ 80-211 Gdansk, Poland \\ Phone/fax: +48 583493910 \\ E-mail: dkozl@gumed.edu.pl \\ bgraff@gumed.edu.pl
}


SNRT (cSNRT) is limited, although marked prolongation of SNRT/CSNRT increases the possibility that sinus node dysfunction may be responsible for syncope [1]. Sinoatrial conduction time (SACT) assessment is not recognized as a diagnostic tool for syncope [2]. It is known that some patients who receive permanent pacemakers due to sinus node dysfunction (SND) do not benefit from it and have syncope recurrences [3]. In some such cases further diagnostic workup leads to the diagnosis of vasovagal syncope (VVS). On the other hand, the only commonly used test for diagnosing vasovagal syncope, the head-up tilt test (HUTT), has many limitations [4]. Moreover, the mechanism of syncope in patients without organic heart disease does not correlate with findings during HUTT $[5,6]$.

The aim of the present study was to identify electrophysiological criteria that can be used to identify patients with sinus node dysfunction and concurrent vasovagal syncope.

\section{Material and methods}

\section{Patients}

Transoesophageal atrial pacing (TAP) was performed in 100 patients. The study group was divided into four groups depending on symptoms and results of TAP:

- group 1: patients with SND and a history suggesting VVS,

- group 2: patients with SND with no previous syncope,

- group 3: patients without SND but with a history suggesting VVS,

- group 4: patients without SND and without a history suggesting VVS.

We used the following diagnostic criteria for sinus node dysfunction:

- clinical features: syncope or presyncope, dizziness, shortness of breath, fatigue, blurred vision, memory loss, chest pain, palpitations [7],
- electrocardiographic features: sinus bradycardia $<50 /$ min, sinus pause $>2 \mathrm{~s}$, sinoatrial exit block [7],

- electrophysiological criteria: corrected sinus node recovery time (cSNRT) > $525 \mathrm{~ms}$ and/or sinoatrial conduction time by Strauss' method (SACT St) $>200 \mathrm{~ms}$ and/or intrinsic heart rate (IHR) lower than predicted $[8,9]$.

The following history findings suggested vasovagal syncope [2]:

- triggers of syncope: prolonged standing, warm environment, injections, sight of blood or syringes, pain, stressful situations,

- concurrent symptoms: diaphoresis, dyspnoea,

- short duration of syncope episodes,

- incidence characteristic: occurrence of different types of syncope suggesting more than one cause of incidents.

Inclusion criteria:

- diagnostic TAP in patients with or without a history of syncope,

- age over 18 years. Exclusion criteria:

- inability to safely discontinue medications which could affect electrophysiological properties of the heart, for at least 5 half-lives prior to TAP (at least 3 months in case of amiodarone),

- contraindications to drugs used in the TAP protocol,

- heart rhythm other than sinus rhythm at the beginning of TAP and HUTT.

The patients had no symptoms of heart, renal, respiratory or hepatic failure. Patients with complex ventricular arrhythmias were not included in the study. The prevalence of coronary artery disease, hypertension and diabetes as well as incidence of syncope and palpitations are shown in Table I. In group 4 there were 3 patients with a history of a single syncope due to an overdose of antihypertensive drugs. They all had a negative HUTT.

Table I. Patients' characteristics

\begin{tabular}{|lcccc|}
\hline Parameter & Group 1 & Group 2 & Group 3 & Group 4 \\
\hline Number of patients & 16 & 24 & 32 & 28 \\
\hline \begin{tabular}{l} 
Age [years]: \\
\hline Mean
\end{tabular} & 45.9 & 63.8 & 43.3 & 52.1 \\
\hline Standard deviation & 21.0 & 15.9 & 18.6 & 18.6 \\
\hline Median (min-max) & $49(18-84)$ & $67.5(26-90)$ & $44.5(18-76)$ & $49.5(18-90)$ \\
\hline Gender: females/males & $10 / 6$ & $18 / 6$ & $23 / 9$ & $18 / 10$ \\
\hline Coronary artery disease, $n(\%)$ & $2(12.5 \%)$ & $1(4.2 \%)$ & $5(15.6 \%)$ & $2(7.1 \%)$ \\
\hline Hypertension, $n$ (\%) & $2(12.5 \%)$ & $1(4.2 \%)$ & $4(12.5 \%)$ & $5(17.9 \%)$ \\
\hline Diabetes, $n(\%)$ & $0(0 \%)$ & $0(0 \%)$ & $1(3.1 \%)$ & $1(3.6 \%)$ \\
\hline Syncope, $n$ (\%) & $16(100 \%)$ & $0(0 \%)$ & $32(100 \%)$ & $3(10.1 \%)$ \\
\hline Palpitations as chief complaint, $n(\%)$ & $2(12.5 \%)$ & $4(16.7 \%)$ & $14(43.8 \%)$ & $18(64.3 \%)$ \\
\hline
\end{tabular}




\section{Study protocol}

The study complies with the Declaration of Helsinki; the protocol of the study was approved by the locally appointed ethics committee. Informed consent was obtained from each patient.

Head-up tilt test was performed in all patients with previous syncope [10]. The test was performed between 8 and 11 am, after an overnight fast. The patient remained supine for $20 \mathrm{~min}$ and then the table was tilted to $60^{\circ}$. An active test with $400 \mu \mathrm{g}$ of nitroglycerine (aerosol, sublingually) was then performed. Passive test lasted $30 \mathrm{~min}$; active test lasted $20 \mathrm{~min}$, or until syncope occurred. In case of contraindications to NTG, a 45 min passive test was performed. The results were interpreted according to 2009 ESC guidelines on management of syncope [2].

Transoesophageal electrophysiological testing was performed in 100 patients [11, 12]. The combination of midazolam and fentanyl was administered to alleviate discomfort related to stimulation. This combination of drugs is safe and does not affect the sinus node function, atrioventricular conduction or the inducibility of tachycardia [13-15].

EDP-1 type 8-point electrodes and a programmable stimulator SP-5 plus (OBREAM - Zabrze, Poland) were used. The Fisher-Bloom EP System and Easy View Plus software (Prosmed, Poland) for personal computer were used for recordings. Pulses of 5 ms width and amplitude up to $20 \mathrm{~mA}$ were delivered. We obtained: SNRT, CSNRT, second pause (IIP), sinoatrial conduction time assessed by Strauss' (SACT St) and Narula's (SACT N) methods and the Wenckebach point (WP).

All the parameters were also assessed after pharmacological autonomic blockade $(\mathrm{AB})$ with propranolol $(0.1 \mathrm{mg} / \mathrm{kg})$ and atropine $(0.02 \mathrm{mg} / \mathrm{kg})$ administered slowly intravenously following the protocol by Wallin [16]. The above protocol is considered safe and efficient in producing a complete autonomic blockade.

Intrinsic heart rate (IHR) was the heart rate recorded after the pharmacological autonomic denervation. Predicted IHR (IHRp) is age-appropriate IHR which is obtained using the formula: IHRp = $118.1 \times(0.57$ - age [years]) [17]. An abnormal value of IHR was defined when it was lower than age predicted by more than $10 \%$.

Sinoatrial conduction time was evaluated by Narula's method using 8 impulses with the rate slightly faster than the basic cycle length $(B C L+$ 10/min) and repeated 3 times [18]. Sinoatrial conduction time was also assessed by the atrial extrastimulus technique [19]. Every eighth sinus cycle (A1 - A1) an extrastimulus (A2) was delivered and the return cycle $(\mathrm{A} 2-\mathrm{A} 3)$ was measured. We used 10 ms decrements until reaching atrial refractoriness. To obtain SACT by Strauss' method the response of sinus node in so-called Zone II [(A1-A3) $<2(\mathrm{~A} 1-\mathrm{A} 1)]$ was considered and the measurements from $1 / 3$ external part of zone II were used.

Sinoatrial conduction time was estimated using the formula: SACT St $=1 / 2[(A 2-A 3)-(A 1-A 1)]$. Sinoatrial conduction time variability was assessed using the following methods:

method (1): SACT max - SACT min,

method (2): (SACT max - SACT min)/SACT max,

(SACT max and SACT min are maximal and minimal values of SACT).

For SNRT, CSNRT and IIP the variability was defined as the difference between maximal and minimal values of the above parameters.

\section{Statistical analysis}

Data are presented as mean \pm SD or percentage as appropriate. Comparison within a group was performed with Student's $t$ test or Mann-Whitney $U$ test. Comparisons between groups were performed by analysis of variance and post-hoc tests. Statistical significance was established at a value of $p<0.05$. All analyses were performed using STATISTICA, version 7.1 (StatSoft, Inc).

\section{Results}

\section{Head-up tilt test}

Group 1: HUTT was positive in $75 \%$ of patients (in 12 out of 16 pts), the pattern being mixed (VASIS type 1$)$ in 8 (66.7\%), cardioinhibitory (VASIS type 2 ) in $3(25 \%)$, and vasodepressor (VASIS type 3 ) in 1 (8.3\%).

Group 3: HUTT was positive in $65.6 \%$ of patients (in 21 out of 32 pts), the pattern being mixed (VASIS type 1) in 14 (66.7\%), cardioinhibitory (VASIS type 2) in 4 (19\%), and vasodepressor (VASIS type 3) in $3(14.3 \%)$ patients.

\section{Transoesophageal atrial pacing}

Transoesophageal atrial pacing was performed in 100 patients. There were no complications. Sinus node recovery time, cSNRT and IIP were successfully obtained in all patients. Sinoatrial conduction time assessed by Narula's method in the basal state was acquired in all patients but it was impossible to assess SACT $N$ after $A B$ in $6 \%$ of patients because of the irregularity of sinus rhythm (in 2 subjects), single premature ventricular or supraventricular beats ( 3 subjects) and the onset of atrial fibrillation before the completion of the study (1 subject). Sinoatrial conduction time assessed by Strauss' in the basal state was obtained in $89 \%$ and after $A B$ in $87 \%$ of patients. Reasons for missing data were lack of so-called Zone II in 4 patients, numerous supraventricular beats in 6 patients, runs 
of AV junctional rhythm in 2 patients and temporary malfunction of the recording device in two subjects.

\section{Standard parameters}

The values of SNRT, cSNRT, IIP, SACT N and SACT St are shown in Table II. After AB, patients in group 1 had significantly shorter SNRT, cSNRT and IIP compared to patients in group 2, while there were no significant differences between patients in groups 1 and 2 concerning SNRT and CSNRT in the basal state. Patients in group 1 also had shorter SACT N and SACT St but the difference was significant only with regard to SACT N. Mean CSNRT, SACT N and SACT St values in group 1 patients shortened after $A B$ and were not significantly different than in groups without SND (3 and 4). All significant differences and the respective $p$-values are shown in Table II.

\section{Intrinsic heart rate}

The values of intrinsic heart rate were $80 \pm 11$, $70 \pm 11,91 \pm 12$ and $90 \pm 15 / \mathrm{min}$ in groups 1 to 4 , respectively. Intrinsic heart rate values were significantly different in group 1 compared to all the other groups (group 1 vs. $2: p=0.016 ; 1$ vs. 3: $p=0.003$; 1 vs. $4: p=0.020)$. Abnormal values of IHR were found in $50 \%$ of patients in group 1 and $54 \%$ in group 2.

\section{Sinoatrial conduction time variability}

In patients with SND and VVS (group 1) SACT St variability was significantly different compared to other groups (Table III). After AB the differences disappeared with the exception of SACT St variability as determined by method (2): (SACT max - SACT min)/SACT max (comparing groups 1 and 2). For method (1): SACT max - SACT min, there was an insignificant trend for greater SACT St variability in group $1(p=0.068)$.

The SACT N variability in patients in group 1 was not different from SACT N variability in patients in other groups. Groups with positive $(A)$ or negative (B) responses to HUTT ( $3 A$ vs. $3 B$ and $1 A+3 A$ vs. $1 B$ $+3 B$ ) did not differ with regard to SACT variability.

\section{SNRT, cSNRT and IIP variability}

In the basal state, the SNRT, CSNRT and IIP variability in patients in group 1 was not different compared to group 2, but there was a significant difference between group 1 patients and patients without SND (group 3 as well as group 4). After AB, the variability of SNRT and CSNRT in group 1 was similar to groups 3 and 4 and significantly different only from group 2. Patients in group 1 had significantly different IIP variability compared to all other patients (Figures 1-3).

\section{Wenckebach point}

In the basal state the values of Wenckebach point were $134 \pm 42,163 \pm 33,156 \pm 27$ and 174 \pm 28 /min in groups 1 to 4 , respectively. After autonomic blockade they were $148 \pm 31,165 \pm 26,166 \pm 21$ and $175 \pm 26 / \mathrm{min}$ in groups 1 to 4 , respectively.

Patients in group 1 had a lower WP than patients in group $4(p=0.003$ in the basal state and $p=0.004$ after $\mathrm{AB})$. There were no statistically significant differences between group 1 and 2 or 3. After correction for age, abnormal WP was found in 6 (37.5\%), 3 (12.5\%), 9 (28\%) and 1 (3.6\%) patient

Table II. Values of SNRT, CSNRT, IIP, SACT N and SACT St and comparison of standard parameters in groups 1-4

\begin{tabular}{|c|c|c|c|c|c|c|c|}
\hline $\begin{array}{l}\text { Sinus node } \\
\text { parameters [ms] }\end{array}$ & Group 1 & Group 2 & Group 3 & Group 4 & $\begin{array}{l}\text { Group } 1 \text { vs. } 2 \\
\text { Value of } p\end{array}$ & $\begin{array}{l}\text { Group } 1 \text { vs. } 3 \\
\text { Value of } p\end{array}$ & $\begin{array}{c}\text { Group } 1 \text { vs. } 4 \\
\text { Value of } p\end{array}$ \\
\hline \multicolumn{8}{|c|}{ Basal state } \\
\hline SNRT & $1579 \pm 578$ & $1652 \pm 310$ & $1151 \pm 213$ & $1178 \pm 242$ & NS & 0.0001 & 0.0011 \\
\hline cSNRT & $613 \pm 412$ & $641 \pm 204$ & $355 \pm 95$ & $345 \pm 101$ & NS & $<0.0001$ & 0.0002 \\
\hline IIP & $1363 \pm 321$ & $1421 \pm 288$ & $927 \pm 279$ & $1009 \pm 285$ & NS & $<0.0001$ & 0.0005 \\
\hline SACT N & $259 \pm 87$ & $236 \pm 63$ & $208 \pm 63$ & $191 \pm 36$ & NS & 0.0171 & 0.0101 \\
\hline SACT St & $193 \pm 42$ & $187 \pm 53$ & $163 \pm 35$ & $166 \pm 30$ & NS & 0.0212 & 0.0456 \\
\hline \multicolumn{8}{|c|}{ Autonomic blockade } \\
\hline SNRT & $1217 \pm 354$ & $2185 \pm 957$ & $953 \pm 168$ & $948 \pm 197$ & $<0.0001$ & 0.0069 & 0.0090 \\
\hline cSNRT & $417 \pm 261$ & $1202 \pm 890$ & $276 \pm 84$ & $267 \pm 84$ & $<0.0001$ & NS & NS \\
\hline IIP & $1002 \pm 295$ & $1748 \pm 913$ & $744 \pm 133$ & $768 \pm 199$ & 0.0002 & 0.0019 & 0.0090 \\
\hline SACT N & $153 \pm 67$ & $222 \pm 115$ & $126 \pm 29$ & $122 \pm 44$ & 0.0180 & NS & NS \\
\hline SACT St & $130 \pm 49$ & $179 \pm 65$ & $118 \pm 23$ & $121 \pm 23$ & NS & NS & NS \\
\hline
\end{tabular}

SNRT - sinus node recovery time, CSNRT - corrected sinus node recovery time, IIP - second pause, SACT N- sinoatrial conduction time by Narula's method, SACT St - sinoatrial conduction time by Strauss' method, NS - non-significant 
Table III. Sinoatrial conduction time by Strauss' method (SACT St ) variability in groups 1-4

\begin{tabular}{|c|c|c|c|c|c|c|c|c|c|c|c|c|}
\hline \multirow[t]{3}{*}{ Method } & \multicolumn{2}{|c|}{$\begin{array}{l}\text { Basal } \\
\text { state }\end{array}$} & \multicolumn{2}{|c|}{$\begin{array}{l}\text { Autonomic } \\
\text { blockade }\end{array}$} & \multicolumn{2}{|c|}{$\begin{array}{l}\text { Basal } \\
\text { state }\end{array}$} & \multicolumn{2}{|c|}{$\begin{array}{c}\text { Autonomic } \\
\text { blockade }\end{array}$} & \multicolumn{2}{|c|}{$\begin{array}{l}\text { Basal } \\
\text { state }\end{array}$} & \multicolumn{2}{|c|}{$\begin{array}{c}\text { Autonomic } \\
\text { blockade }\end{array}$} \\
\hline & \multicolumn{4}{|c|}{ Group 1 vs. 2} & \multicolumn{4}{|c|}{ Group 1 vs. 3} & \multicolumn{4}{|c|}{ Group 1 vs. 4} \\
\hline & 1 & 2 & 1 & 2 & 1 & 3 & 1 & 3 & 1 & 4 & 1 & 4 \\
\hline \multirow[t]{3}{*}{1} & 83.2 & 35.1 & \multirow{3}{*}{\multicolumn{2}{|c|}{$\begin{array}{c}\text { NS } \\
(p=0.068)-\end{array}$}} & 83.2 & 47.8 & \multirow{3}{*}{\multicolumn{2}{|c|}{ NS }} & 83.2 & 32.1 & \multirow{3}{*}{\multicolumn{2}{|c|}{ NS }} \\
\hline & \pm 53.9 & \pm 19.6 & & & \pm 53.9 & \pm 33.6 & & & \pm 53.9 & \pm 22.99 & & \\
\hline & \multicolumn{2}{|c|}{$p=0.004$} & & & \multicolumn{2}{|c|}{$p=0.0291$} & & & \multicolumn{2}{|c|}{$p=0.0009$} & & \\
\hline \multirow[t]{3}{*}{2} & 0.42 & 0.20 & 0.10 & 0.07 & 0.42 & 0.28 & \multirow{3}{*}{\multicolumn{2}{|c|}{ NS }} & 0.42 & 0.19 & \multirow{3}{*}{\multicolumn{2}{|c|}{ NS }} \\
\hline & \pm 0.22 & \pm 0.10 & \pm 0.10 & \pm 0.04 & \pm 0.22 & \pm 0.16 & & & \pm 0.22 & \pm 0.11 & & \\
\hline & \multicolumn{2}{|c|}{$p=0.0031$} & \multicolumn{2}{|c|}{$p=0.0017$} & \multicolumn{2}{|c|}{$p=0.0391$} & & & \multicolumn{2}{|c|}{$p=0.0017$} & & \\
\hline
\end{tabular}

Method (1): SACT max - SACT min, method (2): (SACT max - SACT min)/SACT max, group 1: SND(+) VVS(+), group 2: SND(+), group 3: VVS (+), group 4: SND(-) VVS (-), NS - non-significant

A

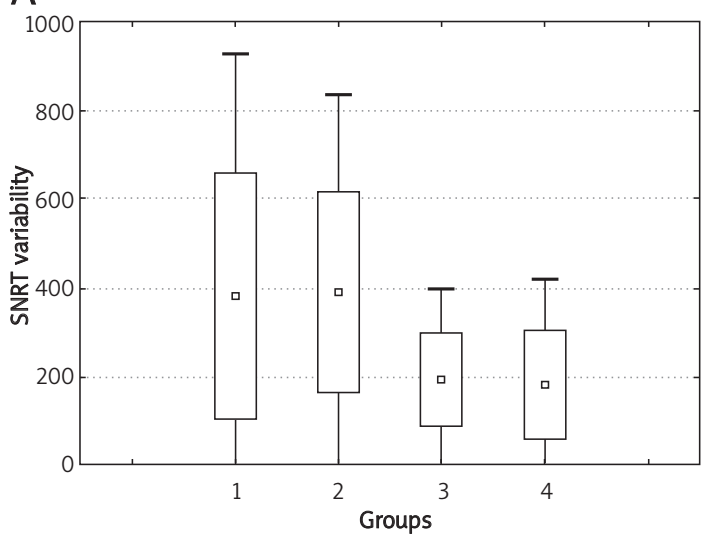

B

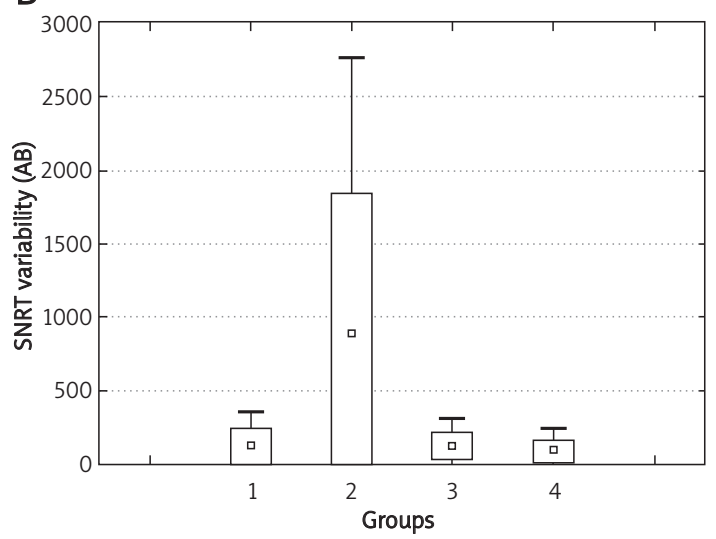

Figure 1. Sinus node recovery time (SNRT) variability in groups 1-4 in the basal state (A) and after autonomic blockade (B)

Group 1: $S N D(+) V V S(+)$, group 2: $S N D(+)$, group 3: VVS (+), group 4: SND(-) VVS (-)

A

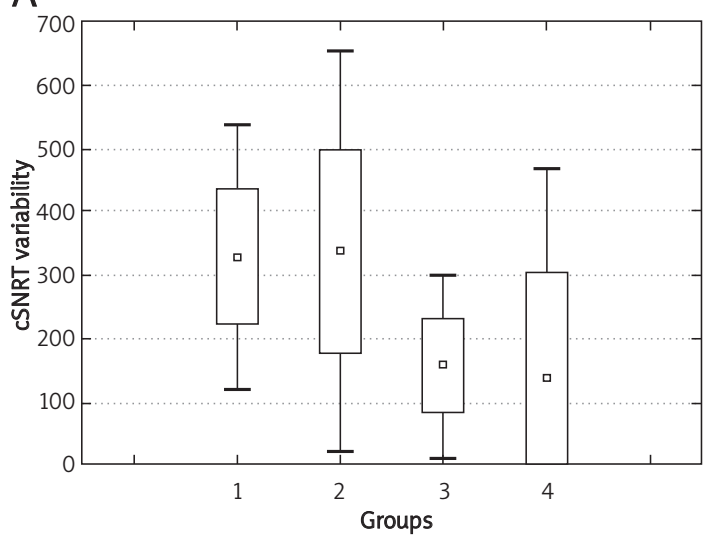

B

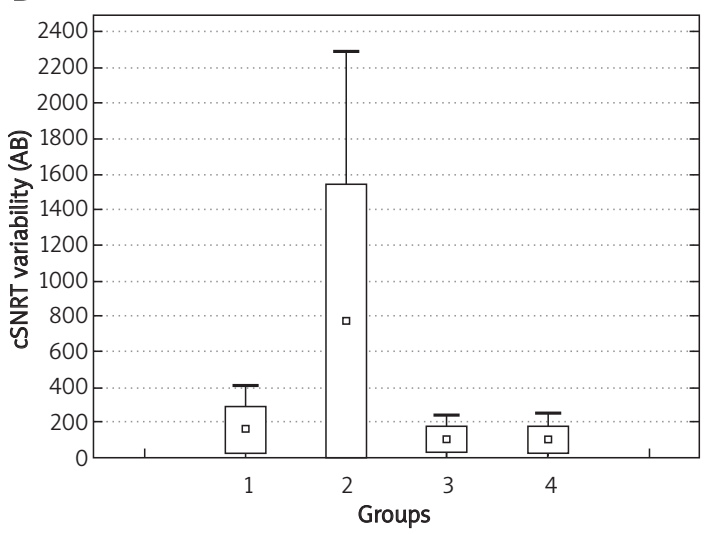

Figure 2. Corrected sinus node recovery time (cSNRT) variability in groups 1-4 in the basal state (A) and after autonomic blockade (B)

Group 1: SND(+) VVS(+), group 2: $S N D(+)$, group 3: VVS (+), group 4: $S N D(-) V V S(-)$

in groups 1 to 4, respectively. Autonomic blockade decreased the number of patients with abnormal WP, especially in patients with VVS. After AB, Wenckebach point below the normal range was found in 5 (31.3\%), 1 (4.2\%), 2 (6.3\%) and 1 (3.6\%) patient in groups 1 to 4 , respectively.

\section{Discussion}

In 1972 Mandel et al. described sinus node recovery time as a new method of assessing sinus node function [20]. After many years the prognostic value of SNRT is still not well defined. Menozzi et al. presented a prospective study in which patients 
A

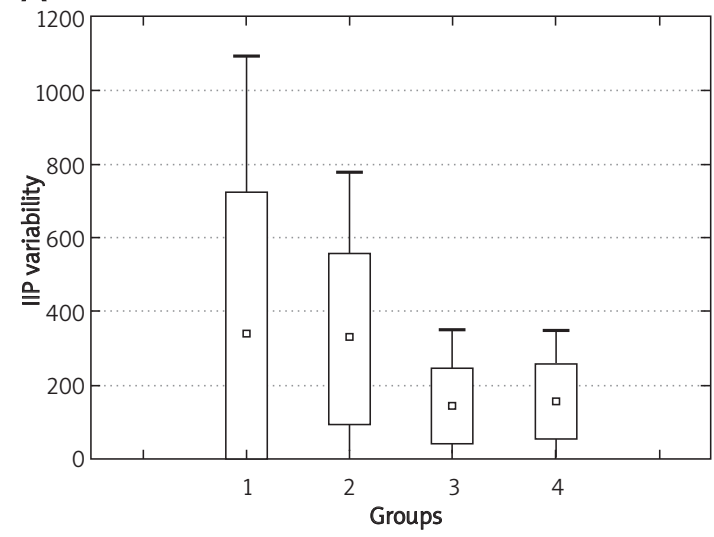

B

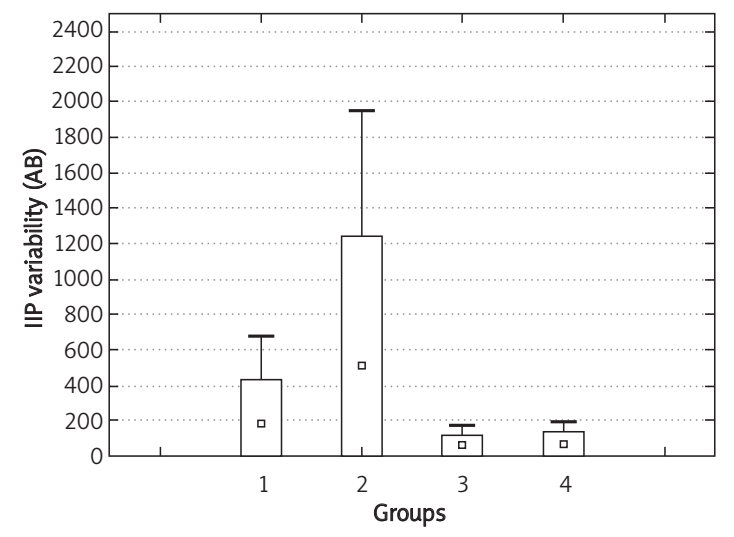

Figure 3. Second pause (IIP) variability in groups 1-4 in the basal state (A) and after autonomic blockade (B) Group 1: SND(+) VVS(+), group 2: SND(+), group 3: VVS (+), group 4: SND(-) VVS (-)

with cSNRT > $800 \mathrm{~ms}$ had an eight times higher risk of having syncope than patients with cSNRTs below this value, but there are no more prospective data on this subject [1]. According to the last ESC Guidelines, prolonged cSNRT > 525 is diagnostic and indicates SND as a cause of syncope [2].

In our study, the basal state values of cSNRT in both groups with SND (1 and 2) were longer than reported by Alboni et al. [21]. After $A B$, the values of CSNRT in group 1 were similar and longer in group 2 patients than in the study cited above.

There were no differences between groups 1 and 2 (with and without syncope) with regard to SNRT, CSNRT and IIP in the basal state. Surprisingly, after $A B$ all these parameters were significantly longer in patients without syncope. Abnormal IHR was found in $50 \%$ of patients in group 1 and $54 \%$ in group 2, so the percentage of intrinsic dysfunction in both groups was the same and cannot be an explanation for different reaction to AB. It appears that patients in group 1 had increased vagal stimulation at the basal state, which is in accordance with the data from heart rate variability analysis in vasovagal patients [22].

There are other studies which also suggest that autonomic modulation plays a crucial role in revealing syncope in patients with SND. Alboni et al. compared two groups of patients with sinus bradycardia less than 50/min [23]. As in our study, cSNRT was not different in patients with a history of syncope and patients without syncope. Patients with incidents of syncope had an abnormal IHR more often (in 66\%) and patients without syncope less often $(26 \%)$ than in our study. Brignole et al. studied 35 patients with SND and incidents of syncope [24]. Fifty-four percent of patients had a positive result of HUTT and in $80 \%$ either HUTT or carotid sinus massage (CSM) were positive. The results were independent of the presence of intrinsic SND or the severity of bradycardia. The authors suggested that an abnormal neural reflex is essential in the development of syncope in SND. This is also supported by reports of results of various ablation procedures on sinus node function in patients treated for tachyarrhythmias $[25,26]$ and "cardioneuroablation" in patients with reflex syncope, functional atrioventricular block and SND [27].

The limitation of the study is that we had no patients with SND and syncope of different than vasovagal origin. Therefore, we are not able to confirm whether group 1 patients really had concurrent vasovagal syncope or they just presented the reflex nature of syncope episodes in SND.

The importance of SACT assessment is still unclear. The Strauss method was the first to estimate SACT and remains the most widely used. It is supposed to be more reliable, but it is time-consuming and has numerous limitations. The method by Narula has an advantage of simplicity but the liabilities are similar to the first method. Based on our previous experience in transoesophageal pacing, we decided to use both methods of SACT assessment to avoid too many missing data.

It has been suggested that the chaotic reaction of the sinus node (SN) to premature impulses is typical for SND. Breithardt et al. have interpreted it as a result of $\mathrm{SN}$ automatism and sinoatrial conduction disturbances [28]. Sadowski and Szwed reported chaotic behaviour of sinoatrial conduction in $8.6 \%$ of patients with SND [29]. They proposed that this chaotic reaction itself indicates SND.

In our study, patients with SND and suspected VVS (group 1) had greater SACT St variability than all other subjects. Additionally, there was no correlation between greater SACT St variability and a positive HUTT result. Thus, increased SACT St variability is the phenomenon characterizing coexistence of SND and VVS. We hypothesize that the main reason for greater SACT variability is the distinct autonomic modulation of a dysfunctional sinus node. Schuessler proposed a new model of the SN based on recent research data [30]. He assumed that the SN consists of cells with different intrinsic rates and that there are specialized pathways 
within the node and a limited number of exit sites. Besides, there is a heterogeneous distribution of autonomic receptors. Such a complex structure may cause different disturbances of sinus rhythm, especially when structural and autonomic changes overlap.

However, there are some technical problems concerning the indirect method of SACT assessment, which is critically dependent on regularity of the sinus cycle. Further studies in larger study groups are needed to ascertain that SACT St variability of sinus rhythm is not just an effect of sinus rhythm irregularity.

The basal state variability of SNRT, CSNRT and IIP in group 1 patients was similar to that in group 2 and significantly greater than the corresponding values in groups 3 and 4 . This may suggest that increased basal state variability of SNRT, CSNRT and IIP is typical for SND. The only explanation we have for greater variability of the above-mentioned parameters in group 2 is the increased sympathetic modulation of the sinus node before $A B$. Once more, an additional group composed of patients with syncope caused by SND alone would possibly facilitate the answer.

Clinical, electrographic and electrophysiological features of SND can be triggered by disturbances of the autonomic nervous system [7]. They can be isolated or concomitant to structural damage of the sinus node. Since Jordan et al.' study, the terms "functional" and "intrinsic" dysfunction of sinus node have been accepted [31]. Assessment of intrinsic heart rate by pharmacological autonomic blockade has become one of the most widely used tests of sinus node function. In our study, pharmacological autonomic blockade was not only the way to detect an intrinsic dysfunction of the sinus node. The $A B$ revealed many additional electrophysiological features, especially in patients with SND and VVS.

During transoesophageal pacing, among various properties of sinus node assessed at baseline and with the use of $A B$, there is also a possibility to test the Wenckebach point, which is considered to reflect atrioventricular (AV) nodal conduction [11]. $W P$ refers to the pacing rate (during incremental atrial pacing) at which AV nodal Wenckebach (Mobitz I) block occurs. In patients with SND the presence of concomitant atrioventricular node dysfunction is important because it affects therapeutic decisions (e.g. the type of implanted pacemaker) [7]. In the present study, patients with SND and VVS had lower WP than patients with neither SND nor VVS. In the basal state, abnormal atrioventricular conduction was more frequent in both groups with VVS. However, it seems that patients with concomitant SND might also have decreased atrioventricular node function which persists after autonomic blockade, indicating organic dysfunction of the AV node. In contrast, subjects with VVS and without SND had mostly functional impairment of AV node conduction, which was due to increased parasympathetic drive.

In conclusion, patients with concurrent sinus node dysfunction and vasovagal syncope have distinct electrophysiological features. This group is characterized by a greater SACT St variability compared to patients without incidents of syncope. Apart from abnormal SNRT, CSNRT and IIP, patients with SND have greater variability of these parameters in the basal state. In patients with SND and concurrent vasovagal syncope the variability of SNRT, cSNRT and IIP decreases after autonomic blockade, indicating increased vagal modulation in the basal state. Transoesophageal atrial pacing is a useful procedure in patients with syncope, especially when the coexistence of more than one cardiac cause is suspected. The protocol after pharmacological autonomic blockade is an essential part of the procedure which can reveal different electrophysiological parameters depending on the distinct aetiology of incidents.

\section{Acknowledgments}

This work was supported by the Foundation of Polish Science TEAM/2008-2/5 (B. Graff) and Polish National Grant no. N N201 373236 (G. Graff).

\section{References}

1. Menozzi C, Brignole M, Alboni P, et al. The natural course of untreated sick sinus syndrome and identification of the variables predictive of unfavorable outcome. Am J Cardiol 1998; 82: 1205-9.

2. The Task Force for the Diagnosis and Management of Syncope of the European Society of Cardiology (ESC). Guidelines for the diagnosis and treatment of syncope (version 2009). Eur Heart J 2009; 30: 2631-71.

3. Sgarbossa EB, Pinski SL, Jaeger FJ, Trohman RG, Maloney JD. Incidence and predictors of syncope in paced patients with sick sinus syndrome. Pacing Clin Electrophysiol 1992; 15: 2055-60.

4. Sheldon R. Tilt testing for syncope: a reappraisal. Curr Opin Cardiol 2005; 20: 38-41.

5. Moya A, Brignole M, Menozzi C, et al. International Study on Syncope of Uncertain Etiology (ISSUE) Investigators. Mechanism of syncope in patients with isolated syncope and in patients with tilt-positive syncope. Circulation 2001; 104: 1261-7.

6. Deharo JC, Jego C, Lanteaume A, Djiane P. An implantable loop recorder study of highly symptomatic vasovagal patients: the heart rhythm observed during a spontaneous syncope is identical to the recurrent syncope but not correlated with the head-up tilt test or adenosine triphosphate test. J Am Coll Cardiol 2006; 47: 587-93.

7. Benditt DG, Milstein S, Goldstein M, Reyes W, Gornick CC. Sinus node dysfunction: pathophysiology, clinical features, evaluation, and treatment. In: Zipes DP, Jalife J. Cardiac electrophysiology: from cell to Bedside. WB Saunders Company, Philadelphia 1990; 708-34. 
8. Narula OS, Shantha N, Vasquez M, Towne WD, Linhart JW. A new method for measurement of sinoatrial conduction time. Circulation 1978; 58: 706-14.

9. Gann D, Tolentino A, Samet P. Electrophysiologic evaluation of elderly patients with sinus bradycardia: a longterm follow-up study. Ann Intern Med 1979; 90: 24-9.

10. Benditt DG, Sutton R. Tilt-table testing in the evaluation of syncope. J Cardiovasc Electrophysiol 2005; 16: 356-8.

11. Santini M, Ansalone G, Cacciatore G, Turitto G. Transophageal pacing. PACE 1990; 13: 1298-25.

12. Levine JH, Kadish AH, Reiter MJ. Transesophageal pacing and recording. W: Zipes DP, Jalife J (ed.). Cardiac electrophysiology: from cell to bedside. WB Saunders Company, Philadelphia 1990; 858-63.

13. Lau W, Kovoor P, Ross DL. Cardiac electrophysiologic effects of midazolam combined with fentanyl. Am J Cardiol 1993; 72: 177-82.

14. Kovoor P, Porter R, Uther JB, Ross DL. Efficacy and safety of a new protocol for continuous infusion of midazolam and fentanyl and its effects on patient distress during electrophysiological studies. Pacing Clin Electrophysiol 1997; 20: 2765-74.

15. Pachulski RT, Adkins DC, Mirza H. Conscious sedation with intermittent midazolam and fentanyl in electrophysiology procedures. J Interv Cardiol 2001; 14: 143-6.

16. Vallin HO. Autonomous influence on sinus node and AV node function in the elderly without significant heart disease: assessment with electrophysiological and autonomic tests. Cardiovasc Res 1980; 14: 206-16.

17. Jose $A D$, Collison $D$. The normal range and determinants of the intrinsic heart rate in man. Cardiovasc Res 1970; 4 : 160-7.

18. Narula OS, Shantha N, Vasquez M, Towne WD, Linhart JW. A new method for measurement of sinoatrial conduction time. Circulation 1978; 58: 706-14.

19. Strauss HC, Saroff AL, Bigger JT Jr, Giardina EG. Premature atrial stimulation as a key to the understanding of sinoatrial conduction in man. Presentation of data and critical review of the literature. Circulation 1973; 47: 86-93.

20. Mandel WJ, Hayakava H, Allen HN, Danzig R, Kermaier Al. Assessment of sinus node function in patients with the sick sinus syndrome. Circulation 1972; 46: 761-9.

21. Alboni P, Paparella N, Cappato R, Pedroni P, Candini GC, Antonioli GE. Reliability of transesophageal pacing in the assessment of sinus node function in patients with sick sinus syndrome. Pacing Clin Electrophysiol 1989; 12: 294-300.

22. Piccirillo G, Naso C, Moise A, et al. Heart rate and blood pressure variability in subjects with vasovagal syncope. Clin Sci (Lond) 2004; 107: 55-61.

23. Alboni $P$, Menozzi C, Brignole $M$, et al. An abnormal neural reflex plays a role in causing syncope in sinus bradycardia. J Am Coll Cardiol 1993; 22: 1130-4.

24. Brignole M, Menozzi C, Gianfranchi L, Oddone D, Lolli G, Bertulla A. Neurally mediated syncope detected by carotid sinus massage and head-up tilt test in sick sinus syndrome. Am J Cardiol 1991; 68: 1032-6.

25. Hocini M, Sanders P, Deisenhofer I, et al. Reverse remodeling of sinus node function after catheter ablation of atrial fibrillation in patients with prolonged sinus pauses. Circulation 2003; 108: 1172-5.

26. Walczak F, Szumowski L, Urbanek P, et al. Successful ablation of focal atrial fibrillation eliminates syncope caused by conduction and automaticity disturbances - reverse remodeling of SA an AV nodes function. Kardiol Pol 2003; 59: 567-73.

27. Pachon JC, Pachon EI, Pachon JC, et al. "Cardioneuroablation": new treatment for neurocardiogenic syncope, func- tional AV block and sinus dysfunction using catheter RF-ablation. Europace 2005; 7: 1-13.

28. Breithardt G, Seipel L, Loogen F. Sinus node recovery time and calculated sinoatrial conduction time in normal subjects and patients with sinus node dysfunction. Circulation 1977; 56: 43-50.

29. Sadowski Z, Szwed H. Significance of premature atrial stimulation in the diagnosis of heart conduction disorders. Kardiol Pol 1983; 26: 637-45.

30. Schuessler RB. Abnormal sinus node function in clinical arrhythmias. J Cardiovasc Electrophysiol 2003; 14: 215-7.

31. Jordan JL, Yamaguchi I, Mandel WJ. Studies on the mechanism of sinus node dysfunction in the sick sinus syndrome. Circulation 1978; 57: 217-23. 